\title{
Re: O'Connor KM and Kiely EA: Lessons learned using Snodgrass hypospadias repair (Irish J Med Sci 175:37)
}

\author{
W. T. Snodgrass
}

Received: 2 May 2007/ Accepted: 26 October 2010/Published online: 9 November 2010

(C) Royal Academy of Medicine in Ireland 2010

To the Editor,

The authors reviewed experience with TIP repair for distal hypospadias, finding $21 \%$ developed meatal stenosis. Unfortunately, they did not describe their postoperative assessment and so the reasons the diagnosis was suspected, means it was established, and time following surgery it occurred are not reported.

As the authors note, meatal stenosis is a well-known complication from hypospadias urethroplasty. However, review of published series on TIP repair indicates this complication occurred in only $1.5 \%$ of patients [1], and in my own experience has been found in less than $1 \%$. I perform a single calibration of the neomeatus 6 months postoperatively to confirm a size $>8 \mathrm{Fr}$ in young boys.

This low incidence of meatal stenosis in contrast to that reported by the authors most likely indicates technical error. The fact stenosis occurred in their first 2, but not the last 2, years performing TIP further reinforces this impression. Furthermore, the authors themselves state technical modifications improved their results. It is worth emphasizing deep incision of the urethral plate and care to avoid tubularizing the neourethra too far distally both decrease likelihood for stenosis. I tubularize the neourethra to approximately $3 \mathrm{~mm}$ from the tip of the glans, and then independently perform glansplasty without securing the neomeatus to the glans wings. In the Introduction, the authors state urethral plate incision should not extend beyond the midglans, but failure to carry the incision further distally to near the end of the plate could contribute to subsequent stenosis.

I have never performed postoperative meatal dilation. The fact less than $5 \%$ of patients are at risk for meatal stenosis calls to question the authors' recommendation for routine dilation for all boys the first months after surgery. While it may be "relatively painless", it is certainly not completely painless and may cause unnecessary anxiety for both the child and his mother.

\section{Reference}

1. Snodgrass WT (1999) Tubularized incised plate hypospadias repair: indications, technique, and complications. Urology 54:6-11

W. T. Snodgrass $(\square)$

Division of Pediatric Urology,

University of Texas Southwestern Medical Center,

Children's Medical Center Dallas,

2350 Stemmons Freeway Suite D-4300,

Dallas, TX 75207, USA

e-mail: warren.snodgrass@childrens.com 